\title{
Reduction in charged defects associated with oxygen vacancies in hafnia by magnesium incorporation: First-principles study
}

\author{
Naoto Umezawa, ${ }^{1, a)}$ Motoyuki Sato, ${ }^{2}$ and Kenji Shiraishi ${ }^{3}$ \\ ${ }^{1}$ National Institute for Materials Science, Advanced Electronic Materials Center, Namiki 1-1, Tsukuba, \\ Ibaraki 305-0044, Japan \\ ${ }^{2}$ Semiconductor Leading Edge Technologies, Inc., Tsukuba 305-8569, Japan \\ ${ }^{3}$ Graduate School of Pure and Applied Sciences, University of Tsukuba, Tsukuba 305-8571, Japan
}

(Received 26 June 2008; accepted 11 November 2008; published online 2 December 2008)

\begin{abstract}
Charged defects in a gate insulating oxide significantly degrade electric properties of the field-effect transistors. We report on our analysis of the effects of $\mathrm{Mg}$ incorporation into $\mathrm{HfO}_{2}$ upon reduction in the positive charges associated with oxygen vacancies $V_{\mathrm{O}}^{+2}$. Our comprehensive study using first-principles calculations revealed that a $\mathrm{Mg}$ atom substituted for $\mathrm{Hf}$ is stable in charge negative $\mathrm{Mg}_{\mathrm{Hf}}^{-2}$ and strongly binds with $V_{\mathrm{O}}^{+2}$, neutralizing the defect. This contributes to the suppressing of the electron traps at the defect site, improving the reliability of Hf-based gate oxides. (C) 2008 American Institute of Physics. [DOI: 10.1063/1.3040306]
\end{abstract}

$\mathrm{HfO}_{2}$ is the most promising high permittivity (high- $\kappa$ ) oxide for the gate dielectric of field-effect transistors (FETs). ${ }^{1,2}$ The positive and neutral oxygen vacancies, $V_{\mathrm{O}}^{+2}$ and $V_{\mathrm{O}}^{0}$, are the predominant defects in $\mathrm{HfO}_{2} \cdot{ }^{3-5}$ This is, however, problematic because the transition from $V_{\mathrm{O}}^{+2}$ to $V_{\mathrm{O}}^{0}$ acts as an electron trap under an applied voltage during the operation of FETs. ${ }^{6}$ Another ionization transition from $V_{\mathrm{O}}^{0}$ to $V_{\mathrm{O}}^{-1}$ has also been recognized as an electron-trapping process in $\mathrm{HfO}_{2}{ }^{7-9}$ The trap and detrap of electrons at $V_{\mathrm{O}}$ causes an instability in the threshold voltage, and thus the suppression of the electron traps is an essential task to improve the reliability of high- $\kappa$ gate oxides.

Previous studies suggested that nitrogen incorporation into $\mathrm{HfO}_{2}$ shifts the $V_{\mathrm{O}}$-induced defect level upward. ${ }^{6,7} \mathrm{~A}$ shallow trap depth is advantageous because any trap electrons could soon be released back to the electrode, suppressing the instability of the FET. Other atomic species have also been incorporated into $\mathrm{HfO}_{2},{ }^{10}$ although most of these works focused on dielectric properties of high- $\kappa$ oxides, and it is rare to find systematic studies for the effects of impurities on reliability issues.

In this letter, we report our extensive study on the effect of $\mathrm{Mg}$ incorporation into $\mathrm{HfO}_{2}$. Our computational results revealed that $\mathrm{Mg}$ is strongly bound with $V_{\mathrm{O}}^{+2}$, neutralizing the defect site.

Our first-principles calculations were based on the density-functional theory (DFT) within the local-density approximation (LDA) using the projector augmented wave pseudopotentials as implemented in the VASP code. ${ }^{11,12}$ The valence configurations of the pseudopotentials are $5 p^{6} 5 d^{2} 6 s^{2}$ for $\mathrm{Hf}, 3 s^{2}$ for $\mathrm{Mg}$, and $2 s^{2} 2 p^{4}$ for $\mathrm{O}$. We used an energy cutoff of $500 \mathrm{eV}$ in the plane-wave basis set expansion. Monkhorst-Pack $k$-point sets of $6 \times 6 \times 6$ and $2 \times 2 \times 2$ were used for a 12-atom unit cell and a 96-atom supercell of monoclinic $\mathrm{HfO}_{2}$ (space group $P 2_{1} / c$ ), respectively. The 12atom cell was used for optimization of the cell parameters, determined as $a=5.04 \AA, b=5.11 \AA, c=5.21 \AA$, and $\beta$ $=99.74^{\circ}$, which are in good agreement with the experimental values $\left[a^{\text {expt }}=5.117 \AA, b^{\text {expt }}=5.175 \AA, c^{\text {expt }}=5.291 \AA\right.$, and

${ }^{\text {a)} E l e c t r o n i c ~ m a i l: ~ u m e z a w a . n a o t o @ n i m s . g o . j p . ~}$ $\beta^{\text {expt }}=99.22^{\circ}$ (Ref. 13)]. The unit cell was extended to the 96-atom supercell to construct models for defects in $\mathrm{HfO}_{2}{ }^{14}$

We explored various possibilities of intrinsic and extrinsic defects with charge $Q$, i.e., a single oxygen vacancy at three-fold and four-fold oxygen sites $\left(V_{\mathrm{O} 3}^{Q}\right.$ and $\left.V_{O 4}^{Q}\right)(Q=0$, +1 , and +2$)$, a $\mathrm{Mg}$ atom substituting for $\mathrm{Hf}\left(\mathrm{Mg}_{\mathrm{Hf}}^{Q}\right)(Q=-2$, -1 , and 0$)$, a $\mathrm{Mg}_{\mathrm{Hf}}$ coupled with an oxygen vacancy at threefold and fourfold oxygen site $\left(\left[\mathrm{Mg}_{\mathrm{Hf}} V_{\mathrm{O} 3}\right]^{Q}\right.$ and $\left.\left[\mathrm{Mg}_{\mathrm{Hf}} V_{\mathrm{O} 4}\right]^{Q}\right)$ $(Q=-2,-1$, and 0$)$, one $\mathrm{Mg}_{\mathrm{Hf}}$ coupled with two $V_{\mathrm{O} 3}$ $\left[\mathrm{Mg}_{\mathrm{Hf}}\left(V_{\mathrm{O} 3}\right)_{2}\right]^{Q}$, and both the $V_{\mathrm{O} 3}$ and $V_{\mathrm{O} 4}\left[\mathrm{Mg}_{\mathrm{Hf}} V_{\mathrm{O} 3} V_{\mathrm{O} 4}\right]^{Q}$ $(Q=-2,-1,0,+1$, and +2$)$. Compensating background charge was introduced for the charged defects to avoid divergence of the total energy. However, we did not apply any corrections to the background charges under the circumstance that there is no reliable scheme at present. The atomic positions were relaxed until the total energy difference was converged within $0.001 \mathrm{eV}$, which results in having the residual forces below $0.03 \mathrm{eV} / \AA$.

The stability of these defects was compared in terms of the formation energy defined by

$$
\begin{aligned}
E_{f}\left(X^{Q}\right)= & E_{\mathrm{tot}}\left(X^{Q}\right)-E_{\mathrm{tot}}(\text { bulk })-\sum_{i} n_{i} \mu_{i} \\
& +Q\left(\varepsilon_{F}+\varepsilon_{v}+\Delta V+\mathrm{E}_{\mathrm{corr}}\right),
\end{aligned}
$$

where $E_{\text {tot }}\left(X^{Q}\right)$ and $E_{\text {tot }}($ bulk) are the total energy of a defect with charge $Q$ and the bulk $\mathrm{HfO}_{2}$, which were given by our DFT calculations. $n_{i}$ and $\mu_{i}$ are the number of atoms deviating from the perfect crystal and the chemical potential of each species $(i=\mathrm{O}, \mathrm{Hf}$, or $\mathrm{Mg})$, respectively. $\varepsilon_{F}$ is the Fermi energy referenced with the valence band maximum $\varepsilon_{v}$ of the bulk $\mathrm{HfO}_{2} . \Delta V$ is the correction to $\varepsilon_{v}$ for the shift in the electrostatic potential due to the introduction of the charged defects into the supercell with respect to that in the bulk $\mathrm{HfO}_{2}$, which we found is of the order of $0.15 \mathrm{eV}$ at a maximum. $E_{\text {corr }}$ is another correction due to the special $k$-point sampling for the shallow acceptor $\left(\mathrm{Mg}_{\mathrm{Hf}}\right.$ in our case): the energy difference between the top of the valence band at $\Gamma$ and the other $k$-points sampled in our calculations, which is about $-0.08 \mathrm{eV}$ for $\mathrm{Mg}_{\mathrm{Hf}}$. We set $\mu_{\mathrm{O}}$ to the condition where a bulk $\mathrm{Si}$ precipitates in $\mathrm{SiO}_{2}$, i.e., $\mu_{\mathrm{O}}=\left(E_{\text {tot }}\left[\mathrm{SiO}_{2}\right]\right.$ 


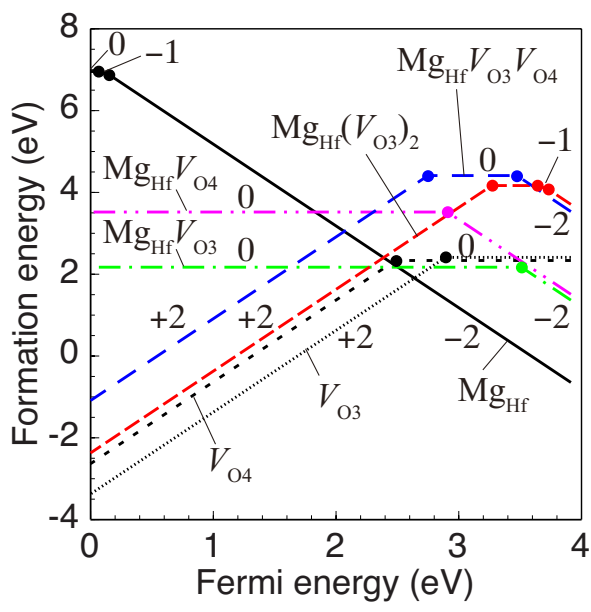

FIG. 1. (Color online) Formation energies of the intrinsic and the Mgrelated defects as a function of the Fermi energy $\varepsilon_{F}$. The zero of $\varepsilon_{F}$ is set to the valence band maximum of bulk $\mathrm{HfO}_{2}$. The number on each line indicates the stable charge state of each defect. The positions of the transition level between different charge states are shown by filled circles.

$-\mu_{\mathrm{Si}}\left[\right.$ bulk])/2 because we are interested in $\mathrm{HfO}_{2}$ deposited on a $\mathrm{Si}$ substrate. Here, the total energy of $\mathrm{SiO}_{2}, E_{\text {tot }}\left[\mathrm{SiO}_{2}\right]$, was obtained from a nine-atom cell for $\alpha$-quartz, and the chemical potential of $\mathrm{Si}$ in a bulk $\mathrm{Si}, \mu_{\mathrm{Si}}[$ bulk], was calculated using the two-atom cell diamond structure. The chemical potential of $\mathrm{Mg}$ was then given by the equilibrium condition $\mu_{\mathrm{Mg}}=E_{\mathrm{tot}}[\mathrm{MgO}]-\mu_{\mathrm{O}}$, where $E_{\mathrm{tot}}[\mathrm{MgO}]$ was calculated from a two-atom unit cell of the rocksalt structure.

In Fig. 1, formation energies of the native and $\mathrm{Mg}$ related defects are shown. Here, the upper limit of $\varepsilon_{F}$ corresponds to the conduction band minimum of $\mathrm{HfO}_{2}{ }^{15} V_{\mathrm{O} 3}^{+2}$ and $\mathrm{Mg}_{\mathrm{Hf}}^{-2}$ predominate in the lower and higher $\varepsilon_{F}$, respectively.

The concentration of a defect directly relates the formation energy, ${ }^{17}$

$$
C\left(X^{Q}\right)=N_{\text {sites }} N_{\text {config }} \exp \left[-\frac{E_{f}\left(X^{Q}\right)}{k_{B} T}\right]
$$

Here, $N_{\text {sites }}$ is the number of sites in the lattice per supercell where the defect can be incorporated and $N_{\text {config }}$ is the number of equivalent configurations on each defect site. $k_{B}$ is Boltzmann's constant and $T$ is the temperature. The Fermi energy of the system is determined by the charge neutrality condition at which the concentrations of dominant defects with positive and negative charges are balanced, namely, $C\left(V_{\mathrm{O}}^{+2}\right)=C\left(\mathrm{Mg}_{\mathrm{Hf}}^{-2}\right)$ in our case. ${ }^{18}$ This gives $\varepsilon_{F}=2.6 \mathrm{eV}$, which corresponds to the crossing point of the two lines of $V_{\mathrm{O}}^{+2}$ and $\mathrm{Mg}_{\mathrm{Hf}}^{-2}$ in the formation energy plots shown in Fig. 1. At this condition, the two complexes $\left[\mathrm{Mg}_{\mathrm{Hf}} V_{\mathrm{O} 3}\right]^{0}$ and $\left[\mathrm{Mg}_{\mathrm{Hf}}\left(V_{\mathrm{O}}\right)_{2}\right]^{+2}$ are relatively stable.

Next, we discuss the temperature dependence of the concentrations of the two stable complexes and their constituents. From Fig. 1, the binding energies of the chemical reactions, $\quad \mathrm{Mg}_{\mathrm{Hf}}^{-2}+V_{\mathrm{O} 3}^{+2} \rightarrow\left[\mathrm{Mg}_{\mathrm{Hf}} V_{\mathrm{O} 3}\right]^{0}$ and $\left[\mathrm{Mg}_{\mathrm{Hf}} V_{\mathrm{O} 3}\right]^{0}+V_{\mathrm{O} 3}^{+2}$ $\rightarrow\left[\operatorname{Mg}_{\mathrm{Hf}}\left(V_{\mathrm{O} 3}\right)_{2}\right]^{+2}$, are calculated from

$$
E_{b}^{(1)}=E_{f}\left(\mathrm{Mg}_{\mathrm{Hf}}^{-2}\right)+E_{f}\left(V_{\mathrm{O} 3}^{+2}\right)-E_{f}\left(\left[\mathrm{Mg}_{\mathrm{Hf}} V_{\mathrm{O} 3}\right]^{0}\right),
$$

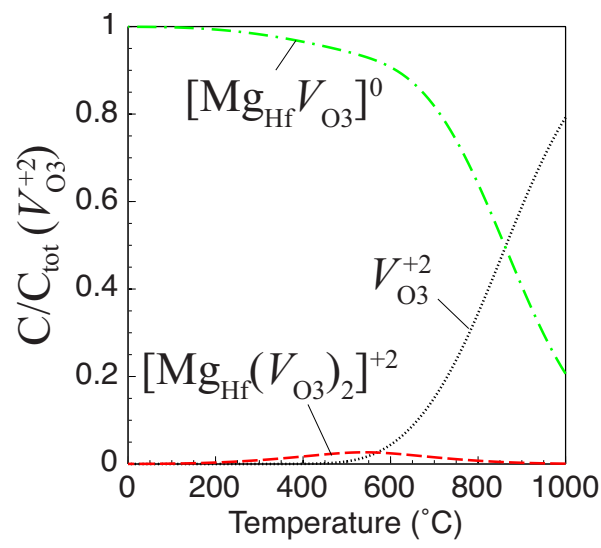

FIG. 2. (Color online) Relative concentration of each defect as a function of temperature.

$$
E_{b}^{(2)}=E_{f}\left(\left[\mathrm{Mg}_{\mathrm{Hf}} V_{\mathrm{O} 3}\right]^{0}\right)+E_{f}\left(V_{\mathrm{O} 3}^{+2}\right)-E_{f}\left(\left[\mathrm{Mg}_{\mathrm{Hf}}\left(V_{\mathrm{O} 3}\right)_{2}\right]^{+2}\right),
$$

which were found to be sizable: $E_{b}^{(1)}=1.6 \mathrm{eV}$ and $E_{b}^{(2)}$ $=1.2 \mathrm{eV}$. The concentrations of the complexes are then given by using $E_{b}^{(1)}$ and $E_{b}^{(2)}$,

$$
\begin{aligned}
& \frac{C\left(\mathrm{Mg}_{\mathrm{Hf}}^{-2}\right) C\left(V_{\mathrm{O} 3}^{+2}\right)}{C\left(\left[\mathrm{Mg}_{\mathrm{Hf}} V_{\mathrm{O} 3}\right]^{0}\right)}=\frac{N_{\text {sites }}}{N_{\text {config }}} \exp \left(-\frac{E_{b}^{(1)}}{k_{B} T}\right), \\
& \frac{C\left(\left[\mathrm{Mg}_{\mathrm{Hf}} V_{\mathrm{O} 3}\right]^{0}\right) C\left(V_{\mathrm{O} 3}^{+2}\right)}{C\left(\left[\mathrm{Mg}_{\mathrm{Hf}}\left(V_{\mathrm{O} 3}\right)_{2}\right]^{+2}\right)}=\frac{N_{\text {sites }}}{N_{\text {config }}} \exp \left(-\frac{E_{b}^{(2)}}{k_{B} T}\right),
\end{aligned}
$$

where $N_{\text {sites }}$ and $N_{\text {config }}$ are 32 and 3, respectively, for both complexes. We solve Eqs. (5) and (6) for each concentration with the condition that

$$
\begin{aligned}
C_{\text {tot }}\left(\mathrm{Mg}_{\mathrm{Hf}}^{-2}\right)= & C\left(\mathrm{Mg}_{\mathrm{Hf}}^{-2}\right)+C\left(\left[\mathrm{Mg}_{\mathrm{Hf}} V_{\mathrm{O} 3}\right]^{0}\right) \\
& +C\left(\left[\mathrm{Mg}_{\mathrm{Hf}}\left(V_{\mathrm{O} 3}\right)_{2}\right]^{+2}\right), \\
C_{\mathrm{tot}}\left(V_{\mathrm{O} 3}^{+2}\right)= & C\left(V_{\mathrm{O} 3}^{+2}\right)+C\left(\left[\mathrm{Mg}_{\mathrm{Hf}} V_{\mathrm{O} 3}\right]^{0}\right) \\
& +2 C\left(\left[\mathrm{Mg}_{\mathrm{Hf}}\left(V_{\mathrm{O} 3}\right)_{2}\right]^{+2}\right)
\end{aligned}
$$

are fixed to those calculated from Eq. (2) for $\varepsilon_{F}=2.6 \mathrm{eV}$ at annealing temperature $T=1000{ }^{\circ} \mathrm{C}$. This implies that the concentration of each defect is in thermodynamic equilibrium under the annealing and then the sample is cooled down in a way that the total number of $\mathrm{Mg}$ atoms and $V_{\mathrm{O} 3}$ is kept fixed. ${ }^{17}$ The results are shown in Fig. 2, which is interpreted as the temperature dependence of the relative concentration of each defect in a same sample. $C\left(V_{\mathrm{O} 3}^{+2}\right)$ is dramatically decreased at lower temperature, thanks to the binding of $V_{\mathrm{O} 3}^{+2}$ with $\mathrm{Mg}_{\mathrm{Hf}}^{-2}$, promoting the formation of the neutral complex $\left[\mathrm{Mg}_{\mathrm{Hf}} V_{\mathrm{O} 3}\right]^{0}$. This results in the predominance of $\left[\mathrm{Mg}_{\mathrm{Hf}} V_{\mathrm{O} 3}\right]^{0}$ at room temperature. Whereas $C\left(\left[\mathrm{Mg}_{\mathrm{Hf}}\left(V_{\mathrm{O} 3}\right)_{2}\right]^{+2}\right)$ is not significantly increased at any temperature. Therefore, $\mathrm{Mg}$ incorporation is very effective for the reduction in the positive charges associated with $V_{\mathrm{O} 3}^{+2}$ at room temperature where the FET is operated.

The large binding energy of $\left[\mathrm{Mg}_{\mathrm{Hf}} V_{\mathrm{O} 3}\right]^{0}$ originates from the attractive Coulomb interactions between $V_{\mathrm{O} 3}^{+2}$ and $\mathrm{Mg}_{\mathrm{Hf}}^{-2}$. We confirmed that the total energy of the neutral $\mathrm{Mg}_{\mathrm{Hf}}-V_{\mathrm{O} 3}$ complex, in which $V_{\mathrm{O} 3}$ is placed at the second nearest neighbor oxygen site to $\mathrm{Mg}_{\mathrm{Hf}}$, is greater than that of $\left[\mathrm{Mg}_{\mathrm{Hf}} V_{\mathrm{O} 3}\right]^{0}$ by $0.95 \mathrm{eV}$, indicating that $V_{\mathrm{O} 3}$ favorably locates near $\mathrm{Mg}_{\mathrm{Hf}}$. 
(a)

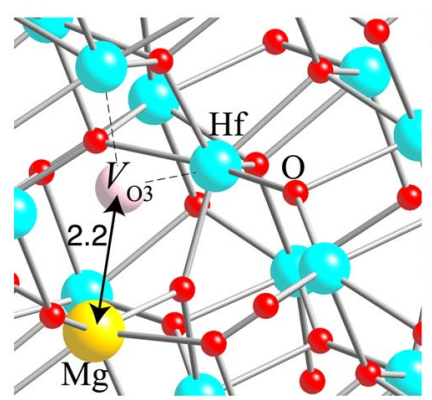

(b)

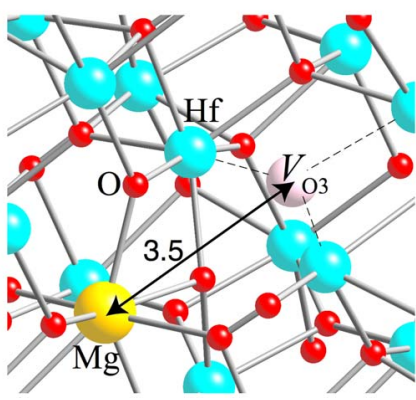

FIG. 3. (Color online) [(a) and (b)] Local geometries of the charge neutral complexes consisting of $V_{\mathrm{O} 3}^{+2}$ and $\mathrm{Mg}_{\mathrm{Hf}}^{-2}$, where $V_{\mathrm{O} 3}^{+2}$ is placed at (a) the nearest neighbor and (b) the second nearest neighbor oxygen sites to $\mathrm{Mg}_{\mathrm{Hf}}^{-2}$, respectively. The distances are in $\AA$.

The local geometries of relaxed structures of these two configurations are depicted in Figs. 3(a) and 3(b) where $\mathrm{Mg}-V_{\mathrm{O}}$ distances are $r_{1}=2.2 \AA$ and $r_{2}=3.5 \AA$, respectively. The difference in the static Coulomb energies between $V_{\mathrm{O} 3}^{+2}$ and $\mathrm{Mg}_{\mathrm{Hf}}^{-2}$ is calculated from

$$
\Delta E=\frac{-4}{\kappa}\left(\frac{1}{r_{2}}-\frac{1}{r_{1}}\right)=0.97 \mathrm{eV},
$$

where the dielectric constant $\kappa=10$ is set smaller than that of the bulk monoclinic $(\kappa \sim 16)$ (Ref. 19) to reflect the insufficient screening effect at the local environment. The estimated value $(0.97 \mathrm{eV})$ is comparable to the total energy difference given by the DFT $(0.95 \mathrm{eV})$, indicating that the Coulomb energy mainly accounts for the attractive interactions.

The decrease in the positively charged defects $V_{\mathrm{O} 3}^{+2}$, which attract negative carriers causing electron traps, can improve the reliability of the gate insulating oxide. The trapped electrons at an isolated $V_{\mathrm{O} 3}$ occupy a deep level in the band gap, which corresponds to the transition level of the charge state from +2 to 0 of $V_{\mathrm{O} 3}, \epsilon(+2 \mid 0)=2.9 \mathrm{eV}$ (Fig. 1). Hence, they are relatively stable and hard to release back to the electrode. On the other hand, the neutral complex $\left[\mathrm{Mg}_{\mathrm{Hf}} V_{\mathrm{O} 3}\right]^{0}$ does not significantly cause the electron traps due to the absence of the attractive interactions between $\left[\mathrm{Mg}_{\mathrm{Hf}} V_{\mathrm{O} 3}\right]^{0}$ and electrons. This effect is reflected in Fig. 1 as an upward shift in the trap level; the transition level of $\mathrm{Mg}_{\mathrm{Hf}} V_{\mathrm{O} 3}$ from 0 to $-2, \epsilon(0 \mid-2)=3.5 \mathrm{eV}$, is much higher than $\epsilon(+2 \mid 0)$ of $V_{\mathrm{O} 3}(2.9 \mathrm{eV})$, indicating that the occupied state associated with $V_{\mathrm{O} 3}$ is destabilized by coupling with $\mathrm{Mg}_{\mathrm{Hf}}$. The electrons occupying such shallow levels are readily released back to the electrode and thus do not significantly degrade the reliability. This is very consistent with the recent experimental reports, ${ }^{20}$ in which $\mathrm{Mg}$ incorporation into the Hf-based high- $\kappa$ dielectric was found to suppress the threshold voltage shift of the FET. Moreover, Mg incorporation may also improve the mobility of the channel current, thanks to the reduction in the positive charges suppressing the Coulomb scattering of the channel carriers.
One may concern that introducing an acceptor such as $\mathrm{Mg}_{\mathrm{Hf}}$ could shift the Fermi level toward the valence band with respect to the intrinsic case, and this results in decreasing the formation energy of $V_{\mathrm{O} 3}^{+2}$ (see Fig. 1), increasing the number of electron trap sites. Even if this is the case at annealing temperature, most of the $V_{\mathrm{O} 3}^{+2}$ are deactivated as electron trap sites by coupling with $\mathrm{Mg}_{\mathrm{Hf}}^{-2}$ at room temperature as discussed before.

In summary, the effects of $\mathrm{Mg}$ incorporation into $\mathrm{HfO}_{2}$ were extensively studied for the reduction in the positive charges associated with $V_{\mathrm{O} 3}^{+2}$. The formation of the neutral complex $\left[\mathrm{Mg}_{\mathrm{Hf}} V_{\mathrm{O} 3}\right]^{0}$ contributes to the suppression of the electron traps in the Hf-based oxides.

We would like to thank T. Chikyow, T. Ohno, K. Yamada, Y. Nara, and J. Robertson for fruitful discussions and advice. N.U. thanks the California Nanosystems Institute (CNSI) for its hospitality. All the calculations were performed on the CNSI computer facilities of Hewlett-Packard clusters.

${ }^{1}$ G. D. Wilk, R. M. Wallace, and J. M. Anthony, J. Appl. Phys. 89, 5243 (2001)

${ }^{2}$ J. Robertson, Rep. Prog. Phys. 69, 327 (2006).

${ }^{3}$ A. S. Foster, F. L. Gejo, A. L. Shluger, and R. M. Nieminen, Phys. Rev. B 65, 174117 (2002).

${ }^{4}$ K. Xiong, J. Robertson, M. C. Gibson, and S. J. Clark, Appl. Phys. Lett. 87, 183505 (2005).

${ }^{5}$ J. X. Zheng, G. Ceder, T. Maxisch, W. K. Chim, and W. K. Choi, Phys. Rev. B 75, 104112 (2007).

${ }^{6}$ N. Umezawa, K. Shiraishi, K. Torii, M. Boero, T. Chikyow, H. Watanabe, K. Yamabe, T. Ohno, K. Yamada, and Y. Nara, IEEE Electron Device Lett. 28, 363 (2007).

${ }^{7}$ K. Xiong, J. Robertson, and S. J. Clark, J. Appl. Phys. 99, 044105 (2006)

${ }^{8}$ J. L. Gavartin, D. M. Ramo, A. L. Shluger, G. Bersuker, and B. H. Lee, Appl. Phys. Lett. 89, 082908 (2006).

${ }^{9}$ P. Broqvist and A. Pasquarello, Appl. Phys. Lett. 89, 262904 (2006).

${ }^{10}$ E. Cockayne, J. Appl. Phys. 103, 084103 (2008), and references therein.

${ }^{11}$ G. Kresse and J. Hafner, Phys. Rev. B 47, RC558 (1993).

${ }^{12}$ G. Kresse and J. Furthmüller, Phys. Rev. B 54, 11169 (1996).

${ }^{13}$ J. Adam and M. D. Rodgers, Acta Crystallogr. 12, 951 (1959); R. E. Hann, P. R. Suttch, and J. L. Pentecost, J. Am. Ceram. Soc. 68, C-285 (1985).

${ }^{14} \mathrm{We}$ confirmed that the 96 -atom supercell is sufficiently large. The formation energy of $\left[\mathrm{Mg}_{\mathrm{Hf}}\left(V_{\mathrm{O} 3}\right)_{2}\right]^{+2}$, which is the largest defect in our calculations, is changed only by $0.08 \mathrm{eV}$ as extending cell size from 72-atom to the 96 -atom supercell.

${ }^{15}$ The computational band gap $(3.9 \mathrm{eV})$ underestimates the experimental value [5.7 eV (Ref. 16)] due to the common problem of LDA. Our conclusion, however, is not significantly affected by this issue because all the thermodynamic transition levels are energetically far distant from the conduction band minimum (Fig. 1) and the corresponding wave functions are well localized at the defect sites (not shown).

${ }^{16}$ H. Takeuchi, D. Ha, and T.-J. King, J. Vac. Sci. Technol. A 22, 1337 (2004).

${ }^{17}$ C. G. Van de Walle and J. Neugebauer, J. Appl. Phys. 95, 3851 (2004).

${ }^{18}$ A. Peles and C. G. Van de Walle, Phys. Rev. B 76, 214101 (2007).

${ }^{19}$ X. Zhao and D. Vanderbilt, Phys. Rev. B 65, 233106 (2002).

${ }^{20}$ M. Sato, N. Umezawa, N. Mise, S. Kamiyama, M. Kadoshima, T. Morooka, T. Adachi, T. Chikyow, K. Yamabe, K. Shiraishi, S. Miyazaki, A. Uedono, K. Yamada, T. Aoyama, T. Eimori, Y. Nara, and Y. Ohji, Dig. Tech. Pap. - Symp. VLSI Technol. 2008, 66. 